\section{CULTURA DE COHESIÓN E INTEGRACIÓN EN LAS CIUDADES CHILENAS ${ }^{1}$}

Guillermo Wormald ${ }^{2}$, Carolina Flores ${ }^{3}$, Francisco Sabatini ${ }^{4}$, María Paz Trebilcock ${ }^{5}$ y Alejandra Rasse Figueroa $^{6}$

\section{Resumen}

Esta investigación plantea que el grado de proximidad espacial y de contacto social entre personas de distinto nivel socioeconómico, moldea las formas de cohesión social, es decir, las normas y valores generadores de actitudes y comportamientos favorables a la supervivencia y desarrollo de una sociedad. La pregunta se centra en las posibilidades para el fortalecimiento de la cohesión social que ofrecen a) las actuales formas de integración y convivencia en los distintos espacios públicos e institucionales en el ámbito urbano, b) las disposiciones a un mayor nivel de contacto social y c) las valoraciones de las oportunidades existentes de contacto. La hipótesis es que una sociedad que ofrece altas posibilidades de contacto entre grupos y que además son altamente valoradas, tendería a ser más cohesiva bajo una lógica menos conflictiva, en la medida en que las personas tomen esas oportunidades.

En una aproximación cualitativa exploratoria de familias de diferente condición social en la Región Metropolitana de Santiago, se observa que la experiencia

\section{COHESIVE CULTURE AND INTEGRATION IN CHILEAN CITIES ${ }^{1}$}

Guillermo Wormald ${ }^{2}$, Carolina Flores ${ }^{3}$, Francisco Sabatini ${ }^{4}$, María Paz Trebilcock ${ }^{5}$ y Alejandra Rasse Figueroa $^{6}$

\section{Abstract}

This research posits that the degree of spatial proximity and social contact among people sharing diverse socioeconomic levels defines social cohesion, that is to say, the norms and values that generate attitudes and behaviors conducive to the survival and development of a society. The main question of this article lies in the possibilities for the strengthening of social cohesion offered by a) current ways of integration and coexistence in different public and institutional spaces within the urban context; $b$ ) willingness to have a greater level of social contact; and c) the value given to the existing opportunities for contact. The hypothesis is that a society offering higher and valued contact opportunities among different groups would show a tendency to be more cohesive in a less conflictive context, so long as people take such opportunities.

An exploratory qualitative approximation to families from different social backgrounds belonging to the Metropolitan Region of Santiago suggests a limited 
que se posee en relación a otros de distinta condición socioeconómica es limitada. La experiencia de heterogeneidad se da mayoritariamente en espacios públicos normados y la expectativa es que este contacto podría ser incómodo y conflictivo. Por ello, la actitud que predomina es la indiferencia. La heterogeneidad aparece como consecuencia de otras decisiones.

\section{PALABRASCLAVE:COHESIÓN SOCIAL;INTEGRACIÓN SOCIAL; CONTACTO.}

Fecha de recepción: 13.07.12

Fecha de aceptación: 24.09.12

1 Este artículo está elaborado en base a lo planteado en el pro yecto de investigación Anillos 2: “Cultura de Integración Socia en las Ciudades Chilenas" (CONICYT SOC-24). El objetivo de este proyecto es indagar acerca de cómo las experiencias de contacto con personas de distinta clase social contribuyen a fortalecer la cohesión social y por ende la sociedad civil.

2 Sociólogo, Doctor en Sociología, Universidad de Sussex, Inglaterra. Profesor Titular de la Pontificia Universidad Católica de Chile.Email: gwormald@uc.cl

3 Socióloga, Pontificia Universidad Católica de Chile. Doctor en Políticas Públicas, Universidad de Austin-Texas, Estados Unidos. Investigadora Instituto de Sociología, Pontificia Universidad Católica de Chile.Email: cfloresc@uc.cl

4 Sociólogo, Pontificia Universidad Católica, Phd. Planificación Urbana, Universidad de California. Profesor e investigador Instituto de Estudios Urbanos y Territoriales, Pontificia Universidad Católica de Chile.Email: fsabatini@uc.cl

5 Socióloga, Universidad Católica, Magíster en Sociología y Candidata a Doctor Instituto de Sociología, Facultad de Ciencias Sociales, Pontificia Universidad Católica de Chile. Investigadora Instituto de Sociología, Pontificia Universidad Católica de Chile. Email: mtrebilc@uc.cl

6 Socióloga, Universidad Católica, Magíster en Sociología, Universidad Católica, Doctor en Arquitectura y Estudios Urbanos, Pontificia Universidad Católica de Chile. Profesor auxiliar, Universidad Católica del Maule.Email: alejandrarasse@gmail.com

118 revista invi № 76 / Noviembre 2012 / Volumen N² 27: 117-145 awareness of the experiences of those of different socio-economic backgrounds.

Such heterogeneity occurs mostly in regulated public spaces, where contact may be expected to be uncomfortable and conflictive. This is why indifference appears as a predominant element. In this case, heterogeneity is the consequence of other decisions.

\section{KEYWORDS: SOCIAL COHESION; SOCIAL INTEGRATION; CONTACT.}

Received: 13.07.12

Accepted: 24.09.12

1 This paper is based on the Anillos 2 research project "Social Integration Culture in Chilean Cities" (CONICYT SOC-24). The goal of this project is to find out how the contact experiences of people from different backgrounds contribute to the strengthening of social cohesion and civil society.

2 Sociologist, $\mathrm{PhD}$ in Sociology, University of Sussex, England. Full Professor, Pontificia Universidad Católica de Chile. Email: gwormald@uc.cl

3 Sociologist, Pontificia Universidad Católica de Chile, PhD in Public Policy, The University of Texas at Austin, United States. Researcher, Instituto de Sociología, Pontificia Universidad Católica de Chile.Email: cfloresc@uc.cl

4 Sociologist, Pontificia Universidad Católica de Chile, PhD in Urban Planning, University of California. Professor and Researcher, Instituto de Estudios Urbanos y Territoriales, Pontificia Universidad Católica de Chile. Email: fsabatini@uc.cl

5 Sociologist, Pontificia Universidad Católica de Chile, MA in Sociology and Doctoral Candidate, Instituto de Sociología, Facultad de Ciencias Sociales, Pontificia Universidad Católica de Chile. Researcher, Instituto de Sociología, Pontificia Universidad Católica de Chile.Email: mtrebilc@uc.cl

6 Sociologist, Pontificia Universidad Católica de Chile, MA in Sociology, Pontificia Universidad Católica de Chile, $\mathrm{PhD}$ in $\mathrm{Ar}$ chitecture and Urban Studies, Pontificia Universidad Católica de Chile. Assistant Professor, Universidad Católica del Maule. Email: alejandrarasse@gmail.com

ARTICLE: Cohesive culture and integration in Chilean Cities / Guillermo Wormald, Carolina Flores, Francisco Sabatini, María Paz Trebilcock y Alejandra Rasse Figueroa 


\section{Introducción}

En estas últimas décadas la sociedad chilena ha vivido profundos cambios en sus características estructurales, lo cual ha ido definiendo diversas formas de contacto entre personas de distinto nivel socioeconómico y por lo tanto en las formas de cohesión social ${ }^{7}$. Un ejemplo de estos cambios es la expansión y movilidad de los sectores medios, especialmente en el ámbito urbano ${ }^{8}$, lo que ha implicado un aumento de la diversidad social y una mayor mixtura en diversos espacios sociales.

A pesar de la creciente movilidad de las clases medias, las clases sociales en los extremos han tendido a replegarse, por ejemplo, en los espacios en que habitan. Diversos autores argumentan que este tipo de repliegue, sobre todo de la pobreza, tiene efectos negativos en varios ámbitos: laboral ${ }^{9}$, educacional $^{10}$, de desarrollo social y emocional ${ }^{11}$, entre otros. El argumento central de esta investigación es que el contacto social entre clases distintas tendría beneficios no sólo individuales, sino además sociales en tanto este contacto puede ser un aspecto central para la generación de comportamientos cohesivos. Así, bajo ciertas condiciones, un mayor grado de proximidad espacial y de contacto social

7 Hopenhayn, 2007.

8 Torche y Wormald, 2004.

9 Wilson, 1997; Sabatini et al, 2008.

10 Jencks y Mayer, 1990; Flores, 2008.

11 Brooks-Gunn, 1992. entre personas de distinto nivel socioeconómico, favorecería la generación de normas y valores que generan actitudes y comportamientos propicios para la supervivencia y desarrollo de una sociedad.

Los argumentos recién presentados sugieren que, aunque las clases en los extremos seguirían estando bastante aisladas del resto, la expansión y mayor movilidad de la clase media proveen oportunidades de contacto social. Pero es posible argumentar que la mera existencia de oportunidades de contacto no es suficiente para que este efectivamente exista, ni tampoco garantiza que el contacto, cuando existe, sea de tipo no conflictivo.

Esta investigación se pregunta por las posibilidades para el fortalecimiento de la cohesión social que ofrecen no sólo las actuales formas de integración y convivencia en los distintos espacios urbanos públicos e institucionales, sino también las valoraciones culturales de las oportunidades existentes de contacto. Estas preguntas son importantes de responder pues tanto las características estructurales de nuestra sociedad actual, como los patrones culturales que significan dichas características, definen los niveles de contacto entre personas de distinto estrato socioeconómico. Por una parte, las características estructurales definen el 
tipo de oportunidades a la que los distintos grupos socioeconómicos pueden optar, dando lugar a espacios más homogéneos o heterogéneos. Por otra parte, los patrones culturales proveen de legitimidad a la distribución de oportunidades, dan significado a dichas oportunidades y generan marcos de interpretación, valoración y comportamiento en las relaciones con personas de otros estratos. Así, la combinación de ambas dimensiones da lugar a determinados niveles de contacto entre personas de distinto estrato socioeconómico (y a determinados espacios físicos de contacto entre distintos).

En resumen, las preguntas que intentan ser abordadas en esta investigación pueden formularse de la siguiente manera:

- ¿Cuál es el concepto del "otro" que emerge en sujetos de diversa condición socioeconómica dentro del ámbito de vida urbana de una sociedad como la chilena?

- ¿Cuáles son las oportunidades de contacto social que ofrece actualmente nuestra sociedad dentro de este ámbito urbano?

- ¿Cómo se valoran estas oportunidades?

- ¿Cuál es la disposición al contacto social entre clases sociales?

120 revista invi № 76 / Noviembre 2012 / Volumen № 27: 117-145
En este artículo expondremos las principales conclusiones que emergen de un estudio cualitativo exploratorio ${ }^{12}$ realizado con familias de diferente condición social en la Región Metropolitana de Santiago. El trabajo se organiza de la siguiente manera. A continuación se presentan algunos conceptos para entender la relación entre cohesión y contacto social. En la tercera parte se analizan las dimensiones objetivas y subjetivas que posibilitan el contacto; en la cuarta parte, se exponen la metodología y los principales resultados que emergen de este estudio. Finalmente se presenta una sección de conclusiones.

\section{Cohesión, contacto social y conflicto}

De acuerdo con el trabajo de Valenzuela ${ }^{13}$, la cohesión social tiene que ver con dos perspectivas fundamentales: a) la de la equidad social o la capacidad de la sociedad para producir una distribución equitativa del poder y del bienestar mediante arreglos institucionales específicamente diseñados para ello, y b) la del fortalecimiento de la sociedad civil o la capacidad de una determinada sociedad de producir normas y valores generadores de actitudes y comportamientos proclives a la confianza

12 Como se expone más adelante, este estudio corresponde a la primera fase de investigación del proyecto Soc-24.

13 Valenzuela, 2008. 
social que permiten generar redes de cooperación entre extraños. Este estudio se enfoca en este segundo aspecto de la cohesión social y entiende que el primero es una variable que afecta a las posibilidades de desarrollo de la sociedad civil.

Esta segunda dimensión responde a la teoría del vínculo social, que ubica la cohesión social en la relación que se da entre los miembros de la sociedad civil y que se basa en planteamientos sobre cooperación, capital social y disposición hacia los otros, que se observan especialmente en la confianza interpersonal, la fortaleza de la asociatividad, la consistencia de las relaciones vecinales y de amistad. En este sentido, lo opuesto a cohesión social es anomia, pero la simple interdependencia funcional no es condición suficiente para generar una sociedad cohesionada; una sociedad moderna des-cohesionada es una sociedad "vacía" de convivencia, asociatividad y sociabilidad con un otro diferente. Una de las dimensiones fundamentales de la cohesión social es la disposición de los individuos a la construcción de patrones de convivencia con un "otro" de condición social diferente y que contribuyen a asentar un sentimiento de cooperación cívica y pertenencia a un todo social.

Además del grado de cohesión social reflejado en las oportunidades de interacción, es necesario distinguir distintos patrones definidos por la posición de los actores en la interacción entre clases.

ARTíCULO: Cultura de cohesión e integración en las ciudades chilenas /

Guillermo Wormald, Carolina Flores, Francisco Sabatini, María Paz Trebilcock y Alejandra Rasse Figueroa
Interacciones basadas en los antiguos patrones hacendales de integración ${ }^{14}$ son cualitativamente diferentes a interacciones entre clases donde los sujetos se relacionan de manera horizontal y conforme a una conciencia y valoración de un fundamento ciudadano. El fortalecimiento de la sociedad civil a través de redes de cooperación y confianza mutua es posible a partir de interacciones legítimas y sostenibles en el tiempo. En esta investigación sostenemos que en el marco de sociedades crecientemente diferenciadas y complejas -como lo es la sociedad chilena hoy-, los patrones culturales asentados en un fundamento ciudadano son más favorables a la generación de interacciones legítimas y durables.

\section{CONTACTO SOCIAL Y CONFLICTO}

Se puede suponer que sin contacto no hay posibilidad de vínculo presencial alguno, lo cual debilita la cooperación y finalmente la cohesión social. Sin embargo, no siempre que se produce contacto se da un vínculo cohesivo. El contacto social puede tener un carácter conflictivo que refuerza una experiencia y valoración negativa del otro. Por ejemplo, cuando las relaciones de confianza se limitan a relaciones con los semejantes, éstas pueden terminar en hostilidad a lo realmente diferente, justo lo opuesto a lo que previamente definimos como fundamento de la cohesión social. La violencia,

14 Medina-Echavarría, 1976. 
la criminalidad y el sentimiento de inseguridad también debilitan la disposición a colaborar y la confianza en el otro ${ }^{15} \mathrm{y}$, por lo tanto, la cohesión social ${ }^{16}$. El aumento del temor conduce a diferentes formas de repliegue y desconexión social que debilitan la consistencia de la sociedad civil.

\section{DEFINICIÓN DEL “OTRO” Y DISPOSICIÓN AL CONTACTO SOCIAL}

Para evaluar el nivel de disposición al contacto con otro diferente, es necesario entender cuál es la imagen que cada clase tiene de las otras. Márquez define el concepto de "imaginario del otro" como aquellas "matrices de sentido que se sitúan en la difusa frontera de lo real y lo imaginado, lo deseado, lo perdido [... Los imaginarios] son cartas de navegación que fundamentan la acción de los sujetos y actores sociales, enriquecen y complejizan la razón, haciéndola deambular entre la realidad y la fantasía"17. El imaginario trata de traer al presente un objeto ausente, recurriendo para esto a la historia. "Los espacios urbanos de lo público, las plazas, las carreteras, los monumentos en la ciudad de la materialidad, veremos que

\footnotetext{
15 Kessler, 2009.

16 Un ejemplo es la violencia criminal en la desorganización de los barrios. "La amenaza más patente a la sociedad civil, sin embargo, proviene de fenómenos de desintegración que provocan de manera muy especial la criminalidad y la violencia social. No es tanto la discriminación, sino el temor la fuente principal de destrucción de la confianza..." (Valenzuela, 2008).

17

Márquez, 2007, p.80.
}

siempre habrá un imaginario que la construye y acompaña. Los imaginarios marcan la ciudady, por ende, la manera de percibirla, de moverse en ella y habitarla"18.

El concepto de imaginario como el aquí presentado es una importante arista en la definición del concepto de "otro". Si el "imaginario del otro" se construye en base a la historia, donde posiblemente la experiencia del otro es algo inexistente (dadas, como veremos en la siguiente sección, la segregación urbana y las nuevas formas de exclusión social), entonces pueden ocurrir dos cosas: la imagen del "otro" puede corresponder a la imagen de un "extraño"19, o bien puede ocurrir que este imaginario -que también expresa lo deseado- refiera a un anhelo de comunidad. Si en el primer caso el concepto del otro debilita la experiencia del otro, en el segundo caso la idealiza bajo la imagen de comunidad.

En relación a la experiencia real del otro y la disposición al contacto, es necesario entender cuales son las condiciones que pone cada grupo para el contacto con otros. Por esto, este estudio se pregunta por los umbrales de tolerancia que establecen las

18 Ibid

19 Sennet, 1978 
clases sociales a la convivencia con un otro diferente en los diversos espacios urbanos que estructuran la vida en la ciudad (barrio, escuela, espacios públicos, trabajo). Por lo tanto, no hablamos de cohesión social en un sentido amplio -o a escala nacional- sino más bien de aquellos elementos que posibilitan o dificultan la disposición y el encuentro con otro de condición social diferente en estos distintos espacios.

La cohesión social entendida como la disposición de los individuos a la construcción de patrones de convivencia con un "otro" de condición social diferente y construido desde un imaginario favorable de dicha convivencia ${ }^{20}$, depende de dos dimensiones: una objetiva, que dice relación con las oportunidades de integración y la experiencia del otro, y una subjetiva, que se refiere a las valoraciones culturales de las formas de integración, experiencia y vínculo social. La siguiente sección se refiere a cada una de estas dimensiones.

\section{Oportunidades de contacto y valoración de éstas}

La sociedad chilena ha sufrido un cambio significativo en las últimas tres o cuatro décadas. A las consecuencias de los cambios en el modelo de acumulación -que se inicia en 1973 y se mantiene

20 Márquez, 2007. vigente hasta hoy- se añade una transformación en los valores predominantes, por la cual la sociedad chilena se ha ido transformando crecientemente en una sociedad de mercado ${ }^{21}$. A continuación se exponen brevemente los aspectos relevantes (para esta investigación) de estas transformaciones, especificando en cada caso las consecuencias de estos cambios para las oportunidades de contacto social. Un segundo apartado de esta sección se refiere a un tema de carácter más subjetivo, que tiene que ver con los ejes conceptuales necesarios para orientar el estudio de la disposición al contacto social.

\section{TRANSFORMACIONES AL MODELO DE ACUMULACIÓN Y OPORTUNIDADES DE CONTACTO}

El nuevo modelo de acumulación conlleva dos fenómenos estrechamente relacionados. En primer lugar, la estructura oportunidades se ha segmentado de manera ad-hoc a la condición socio-económica de cada cual, lo que ha generado una disminución de contacto entre actores de diferente condición social en los espacios asociados a estas oportunidades. En segundo lugar, los distintos espacios de uso cotidiano en la ciudad, tales como el barrio, la escuela y algunos espacios públicos, tenderían hacia una mayor homogeneidad en términos de sus

21 Slater y Tonkins, 2001. 
características socioeconómicas ${ }^{22}$. Ambos fenómenos están estrechamente relacionados en tanto que la segmentación de las oportunidades según grupo socioeconómico lleva a la distribución desigual de las oportunidades en el territorio. De este modo, algunas áreas más interesantes para el mercado concentran diversos usos y oportunidades, y otras quedan relegadas. Esto genera un nuevo ciclo de desigualdades, no sólo por la mayor dificultad para acceder a las oportunidades más lejanas, sino por el surgimiento de fenómenos de deterioro urbano en aquellos sectores más desaventajados ${ }^{23}$.

El nuevo modelo de acumulación ha generado características estructurales en las ciudades que han hecho que la experiencia social cotidiana de una gran parte de las personas incluya sólo a otros de igual condición social. La ciudad -como agencia de la experiencia urbana- iría perdiendo la riqueza de su diversidad. Esto es preocupante, considerando que las potencialidades de la ciudad surgen precisamente de la interrelación de lo diverso ${ }^{24}$. Adicionalmente, la falta de experiencia efectiva de contacto con otros de distinta condición social tiene efectos, tanto en la capacidad de formación de capital social y comunitario, especialmente de las personas más pobres, como también en su

22 Kaztman, 2001

23 Harvey, 1973.

24 Lefebvre, 1969.

124 revista invi № 76 / Noviembre 2012 / Volumen N ํ 27: 117-145 capacidad de conocimiento y acceso a las oportunidades de la ciudad ${ }^{25}$.

Podemos mencionar al menos dos aspectos que tienden a mitigar estos fenómenos de segmentación de oportunidades y de segregación urbana. Por un lado, a pesar de las transformaciones neoliberales, el Estado ha seguido siendo un proveedor fundamental de protección y seguridad social para amplios segmentos de la sociedad, lo que impide que el grupo más pobre quede totalmente desconectado del resto de la población. Por otro lado, existen estudios que dan cuenta de una reducción de la escala de la segregación residencial ${ }^{26}$, lo que de alguna manera ha acercado a las clases medias y bajas.

Las oportunidades de integración y experiencia del otro se dan en los espacios públicos institucionales que estructuran la vida cotidiana de las personas en la ciudad. Nuestro estudio identifica cuatro espacios principales: el barrio; la escuela; el trabajo y los espacios públicos recreativos, comerciales y de transporte. Estos espacios encierran formas de convivencia y valoraciones culturales que especifican, fortalecen o debilitan la cohesión social.

25 Sabatini, Salcedo, Wormald y Cáceres, 2010

26 Sabatini et al, 2005 y 2008 para el caso de Santiago.

ARTÍCULO: Cultura de cohesión e integración en las ciudades chilenas / Guillermo Wormald, Carolina Flores, Francisco Sabatini, María Paz Trebilcock y Alejandra Rasse Figueroa 
El barrio: la encuesta Ecosocial ${ }^{27}$ reporta una percepción bastante alta de apertura vecinal (el 37\% clasifica a los vecinos en la misma posición que la propia, un 39\% los clasifica en una escala superior y el restante $24 \%$ en una posición inferior). Por otra parte, el barrio parece tener una valoración diferencial por estrato socio-económico. Mientras para la población de menores recursos, el barrio es importante como fundamento de vínculo social, para los sectores medios y altos la valoración del barrio -sin dejar de ser ámbito de convivencia- es más como símbolo de status e inversión en renta urbana.

La escuela: cuando se elige, es un ámbito que tiende a la convivencia entre iguales. Se tolera limitadamente la presencia de otro de condición social inferior. Se valora como una experiencia de diversidad, pero desde un ámbito mayoritario de igualdad. Lo importante, más allá de la calidad de la educación, son las redes sociales (vínculos débiles) que posibilita.

El trabajo: la encuesta sobre trabajo y equidad, 2008 concluye que Chile no es un país polarizado. Las relaciones laborales son satisfactorias o

27 Esta encuesta, realizada el año 2007, estudió a 7 países de A.L., incluido Chile, y se encuestó a los habitantes de 18 años y más, de ambos sexos, con nacionalidad del país, pertenecientes a todos los niveles socio-económicos de las principales ciudades: Gran Santiago, Valparaíso, Viña, Antofagasta, Concepción, Talcahuano, Temuco, La Serena y Coquimbo. Los resultados de esta encuesta están compilados en Valenzuela, 2008. muy satisfactorias. Las relaciones entre sindicato y empresa según los trabajadores encuestados son cooperativas y no conflictivas, las relaciones con sus pares es buena $(82,4 \%)$ y también es óptima la relación con sus jefes directos (82,2\%). Menos positiva es con los dueños de la empresa (47\%). Adicionalmente, los trabajadores tienen confianza en que la empresa va a cumplir sus promesas (67\%). Agreguemos que según nuestros datos, el trabajo se constituye en un espacio central para el desarrollo de vínculos de sociabilidad entre los trabajadores.

Espacios públicos recreacionales, comerciales y de transporte: en tanto ámbitos des-institucionalizados existe poca evidencia o datos empíricos, no obstante las preguntas relevantes serían: ¿qué tipo de experiencia del otro potencian? ¿Son espacios capturados por la segmentación, el temor y la desconfianza hacia el otro, o se constituyen como una experiencia positiva de ciudadanía con el otro? Nuestros datos permiten afirmar que, en general, en espacios públicos socialmente heterogéneos tienden a predominar actitudes de indiferencia y/o tolerancia hacia los otros. El temor más 
generalizado es a la falta de respeto de ese otro que se lo percibe como un total desconocido que desafía las normas y valores comúnmente aceptados: el "flaite".

\section{UN CAMBIO EN LOS VALORES DE LA SOCIEDAD}

Un cambio en los patrones culturales de la sociedad que pasa a una matriz más individualista, afecta los niveles de contacto entre personas de distinto estrato socioeconómico. Asimismo, estos patrones que proveen de legitimidad a la distribución de las oportunidades generan marcos de interpretación, valoración y comportamiento en las relaciones con personas de otros estratos. En este sentido, los patrones culturales pueden cambiar la segmentación de la estructura de oportunidades, afectando su legitimidad. Por otra parte, el contacto efectivo con personas de distinta situación económica puede, a su vez, ir modificando paulatinamente la forma en que se conciben las relaciones entre personas de distintos estratos, la valoración de la homogenei$\mathrm{dad} /$ heterogeneidad, e incluso la legitimidad de la forma de acceso a las oportunidades.

En el caso de una sociedad como la nuestra, junto con estos cambios en la estructura de oportunidades y experiencia de vida en la ciudad, la sociedad

126 revista invi № 76 / Noviembre 2012 / Volumen № 27: 117-145 chilena ha comenzado a transitar hacia una sociedad en la cual las identidades, valoraciones y expectativas -especialmente de los nuevos grupos medios- se identifican con los valores de una sociedad de mercado ${ }^{28}$. Por ejemplo, la encuesta Ecosocial ${ }^{29}$ muestra que 2 de cada 3 chilenos, independiente de su clase social, están de acuerdo con la idea de que es el individuo quien debe buscar oportunidades de bienestar. Asimismo, la encuesta Trabajo y Equidad ${ }^{30}$ muestra que el grueso de la población -sobre todo los jóvenes- cree que son las características personales, como la iniciativa y talento personal, flojera y falta de iniciativa, responsabilidad y trabajo duro, vicios y alcoholismo, las que más inciden en el futuro de una persona. En tanto, un 45\% cree que debería haber mayores premios al esfuerzo individual y un 58\% estima que es tarea de cada uno buscarse oportunidades $^{31}$. Una tercera fuente de información, la encuesta Bicentenario, muestra resultados similares. Según esta encuesta, el 65\% está de acuerdo con que el trabajo, el esfuerzo y la iniciativa personal son los principales recursos para mejorar la condición de vida de cada cual. Un $60 \%$ piensa que el éxito económico depende del esfuerzo personal y que la remuneración debe atarse a la productividad de cada cual. Adicionalmente, un $68 \%$ piensa

28 Slater y Tonkins, 2001

29 Valenzuela, 2008.

30 Consejo Asesor Presidencial Trabajo y Equidad, 2008.

31 Ibíd.

ARTÍCULO: Cultura de cohesión e integración en las ciudades chilenas / Guillermo Wormald, Carolina Flores, Francisco Sabatini, María Paz Trebilcock y Alejandra Rasse Figueroa 
que éstos son los principales recursos para superar la pobreza. Por otra parte, desde el 2006 hasta hoy existe un marcado optimismo acerca del desarrollo económico del país y las oportunidades de movilidad social ${ }^{32}$.

Todos estos antecedentes dan cuenta del fenómeno de individuación de la vida social, lo cual ha ido acompañado por altas expectativas -especialmente entre los jóvenes- y un proceso efectivo de movilidad social. La encuesta Ecosocial señala que las expectativas de movilidad social son altas, especialmente entre los jóvenes: "casi el 70\% de la población considera que ascenderá socialmente en los próximos 10 años y más del 80\% espera que los hijos mejoren su posición social". Es interesante consignar, que en estos porcentajes también están incluidos los segmentos de menores recursos, quienes son igualmente optimistas acerca de sus oportunidades de movilidad social. Asimismo, un 43\% de los entrevistados espera lograr una posición social más alta que la de sus padres, y un 40\% declara haber ascendido en la escala social en los últimos 10 años (versus un 30\% que declara haber descendido).

32 Universidad Católica y Adimark, Encuesta Bicentenario, 2009 La publicación del año 2009 compara la serie desde el año 2006 al 2009.
Datos de esta misma encuesta Ecosocial permiten observar que para un segmento no despreciable de la clase media urbana (87\%), el progreso e integración a las oportunidades de vida se relacionan con las nuevas oportunidades educacionales, la iniciativa personal y el trabajo duro. Esto es consistente con los hallazgos de Torche y Wormald ${ }^{33}$ que muestran que los patrones de movilidad social efectivamente experimentada por las diversas categorías de trabajadores tienen que ver con criterios adscritos y adquiridos como la educación. Sin embargo, en la encuesta Trabajo y Equidad, 2008, al testear las preferencias entre la opción de más igualdad o más crecimiento, hay una clara preferencia por una mayor igualdad. Un $65 \%$ opta por más igualdad, incluso a costa de un menor crecimiento ${ }^{34}$. Ello puede estar reflejando un sentimiento y valoración de una mayor solidaridad social. ${ }^{35}$ Así, se observa que la sociedad chilena aún se mueve entre la adscripción y el logro, lo que abre la pregunta por los patrones de cohesión social existentes en una sociedad como la nuestra. Los recientes procesos de individuación y acceso masivo a la educación media y superior han ido acompañados de una reivindicación ciudadana por más

33 Torche y Wormald, 2004

34 Consejo Asesor Presidencial Trabajo y Equidad, 2008, pág.32-33.

35 Por otra parte, no obstante los nuevos valores de mercado, un $65 \%$ piensa que el Estado debería incrementar sustantivamente el control de la economía (Ibíd, p. 34) lo que puede estar reflejando una valoración de los esfuerzos estatales orientados hacia la provisión de protección social. 
y mejores oportunidades de vida, lo que da cuenta de que la sociedad chilena hoy aspira a una mayor equidad y a una ciudadanía más completa.

Los antecedentes presentados dan cuenta de un proceso de transformación en las oportunidades de vida y valoración social que afectan las formas de convivencia, la naturaleza del vínculo social y las valoraciones culturales de los habitantes urbanos. En el caso de los sectores más pobres, este fenómeno puede redundar en un mecanismo de exclusión y anomia. Y en el caso de los sectores más ricos, en una experiencia empobrecida de la ciudad y un imaginario del otro marcado por prejuicios, miedo y/o desconfianza que termina por un abandono "de la ciudad, en la ciudad".

\section{Metodología}

El proyecto Anillos "Cultura de Integración y cohesión social en ciudades chilenas" tiene tres fases empíricas. Una primera fase exploratoria de tipo cualitativa, una segunda fase que incluye una encuesta (construida en base a los resultados de la primera fase) y una tercera fase cualitativa que profundiza en los resultados de la encuesta.

Los resultados que se exponen en este trabajo corresponden a esta primera fase cualitativa. El objetivo principal de esta fase fue conocer las experiencias efectivas de contacto entre personas de distinta clase social, el significado entregado a esas experiencias y la disposición a tener mayores contactos con otros de clase social diferente. El resultado de esta fase fue el desarrollo de indicadores que pueden ser medidos en la segunda fase cuantitativa de la investigación.

En esta primera fase se desarrollaron entrevistas en profundidad a jefes de hogar de diferente grupo socioeconómico en la ciudad de Santiago. Las entrevistas fueron realizadas en la modalidad de una conversación abierta en base a tópicos previamente definidos en una pauta temática que trataba: los imaginarios de las clases sociales, la experiencia de contacto con personas de otra clase en distintos espacios y la disposición al contacto con otros de distinta clase social.

La muestra se segmentó de acuerdo a una tipología de espacios educativos y barriales, en función de su nivel de homogeneidad/heterogeneidad social y de su nivel socioeconómico (alto, medio y bajo). El supuesto que está detrás del uso de este criterio de segmentación es que la experiencia efectiva de heterogeneidad social que tienen las personas, especialmente cuando ellas optan por estos espacios 


\section{TABLA 1: SEGMENTACIÓN DE LA MUESTRA.}

\begin{tabular}{l|ccc}
\hline Tipo de barrio según homogeneidad y clase social & \multicolumn{3}{c}{ Tipo de establecimiento según homogeneidad y clase social } \\
Homogénea alta & Heterogénea & Homogénea baja \\
\hline Homogéneo alto & 2 & 1 & \\
Homogéneo medio & 2 & 3 & 1 \\
\hline Homogéneo bajo & & 1 & 1 \\
Heterogéneo alto & 2 & 2 & 2 \\
Heterogéneo medio & 2 & 1 & \\
Heterogéneo bajo & & 9 & 2 \\
\hline Total & 8 & & \\
\hline
\end{tabular}

heterogéneos, cambia el imaginario y la percepción que se tiene del otro. ${ }^{36}$

El cruce de la variable de nivel socioeconómico del hogar y heterogeneidad de los espacios barriales y educacionales escogidos por el hogar genera una muestra que comprende una diversidad de situaciones. De este modo, se consideraron distintos tipos de familias:

36 La clasificación del nivel de homogeneidad/heterogeneidad social se realizó en base a la dispersión de ingresos en la zona censal (para el caso del barrio) y de la dispersión del nivel socioeconómico reportado en la escuela (tomando como base el Simce). Cabe señalar que esta asimilación de una zona censa con la noción de barrio es un recurso metodológico necesario para seleccionar la muestra, que no intenta dar cuenta de la riqueza del concepto de barrio propiamente tal. Este último emerge en la apreciación de nuestros entrevistados con límites diversos especificando, sin embargo, una experiencia de vecindad claramente identificada.

La selección final del entrevistado en cada uno de los barrios se complementó con información de nivel educacional y ocupación del jefe de hogar, de manera de asegurar que el caso correspondiese al NSE.

ARTÍCULO: Cultura de cohesión e integración en las ciudades chilenas /

Guillermo Wormald, Carolina Flores, Francisco Sabatini, María Paz Trebilcock y Alejandra Rasse Figueroa
- familias que afirman su identidad social en la elección de barrio y colegio o liceo (escogiendo en ambos casos espacios homogéneos);

- familias que valoran la heterogeneidad (o al menos le dan menos peso que a otros atributos del barrio y del colegio o liceo); 
- familias que están haciendo un esfuerzo de movilidad social (poniendo a sus hijos en establecimientos con alumnos de mayor nivel socioeconómico) y

- familias que, por no tener la capacidad de pago para escoger, han quedado encerradas en espacios homogéneos de estratos bajos.

Se decidió tomar familias en este ciclo de vida (padres con hijos en edad escolar) porque ellos han tomado una decisión de localización relativamente definitiva, tanto en el barrio como en la escuela.

Esta combinatoria no sólo da una diversidad de tipos posibles, sino una tipología de familias de acuerdo a su nivel socioeconómico, su situación en términos de movilidad y su valoración de la heterogeneidad social en relación a otros atributos. La muestra total quedó conformada de la siguiente manera:

En total se realizaron 19 entrevistas. La información fue analizada con el software Nvivo8. El trabajo de campo fue realizado entre noviembre y diciembre del 2009. Dentro de cada espacio seleccionado, se escogió al jefe de hogar de edad media (30 a 45 años).

\section{Resultados}

Se observa que existe una dificultad importante para poder identificar elementos unificadores que permitan establecer una imagen en relación al significado de ser chileno. En general, los entrevistados apuntan a un imaginario/experiencia de diversidad que se relaciona inmediatamente al ordenamiento de la sociedad en base a estratos socioeconómicos. Esto lleva a que la percepción de los individuos acerca de la sociedad chilena ponga más énfasis en las diferencias que en las similitudes, remitiendo de manera inmediata a los criterios de distinción que articulan el orden social en estratos socioeconómicos o clases.

De acuerdo a los entrevistados, el principal criterio de estratificación y pertenencia a una clase social es el ingreso (expresado también como consumo o capacidad de pago). Al interior de las clases definidas por el ingreso, se añaden una serie de diferencias de orden cultural.

"(Respecto a la clase media) pero yo no estoy hablando
de esa clase, te estoy hablando de gente que está en los
mismo colegios que nosotros y todo, pero que no les sobra
la plata. A eso me refiero... Y luego, hay una clase defi-
nitivamente rica. O sea, que tienen, no sé pos, segunda
vivienda, un auto para cada uno" (Matías, alto, barrio
homogéneo, escuela homogénea).

Los entrevistados logran percibir entre 2 y 5 clases sociales. La capacidad de realizar distinciones de clases sociales está aparentemente relacionada con el nivel educacional (capital cultural de las personas) y la proximidad con la clase (mientras más cercana, más distinciones se realizan, de manera 


\section{TABLA 2: CRITERIOS DE CLASIFICACIÓN DE CLASES SOCIALES.}

\begin{tabular}{|c|c|c|c|}
\hline & Clase alta & Clase media & Clase baja \\
\hline $\begin{array}{l}\text { Características del } \\
\text { jefe de hogar }\end{array}$ & $\begin{array}{l}\text { Son profesionales, dueños de } \\
\text { empresa y altos ejecutivos. } \\
\text { Además trabajan en algo que } \\
\text { les gusta. }\end{array}$ & $\begin{array}{l}\text { Compuesta por profesionales } \\
\text { o trabajadores calificados } \\
\text { que han surgido en base al } \\
\text { esfuerzo y trabajo personal. } \\
\text { El acceso a un trabajo estable } \\
\text { les permite asegurar ingresos } \\
\text { y capacidad de consumo. }\end{array}$ & $\begin{array}{l}\text { No tienen profesión, por ende, } \\
\text { sus trabajos tienden a ser de } \\
\text { mala calidad y desprotegidos. } \\
\text { También se asocia a situacio- } \\
\text { nes de empleos esporádicos y } \\
\text { cesantía. }\end{array}$ \\
\hline Capacidad de pago & $\begin{array}{l}\text { No les cuesta darse lujos. } \\
\text { Viajan al extranjero por placer, } \\
\text { tienen servicio doméstico, } \\
\text { tienen más de un auto, tienen } \\
\text { segunda vivienda. }\end{array}$ & $\begin{array}{l}\text { No pasan necesidades, pero } \\
\text { tienen que esforzarse para } \\
\text { mantener su nivel de vida. }\end{array}$ & $\begin{array}{l}\text { No les alcanza para cubrir sus } \\
\text { necesidades básicas. La esca- } \\
\text { sez de recursos es lo que les } \\
\text { impide surgir. }\end{array}$ \\
\hline Acceso a servicios & $\begin{array}{l}\text { No usan transporte público. } \\
\text { Usan servicios privados. } \\
\text { Van a colegios particular } \\
\text { pagados y pagan por salud. }\end{array}$ & $\begin{array}{l}\text { Aunque les cueste, prefieren } \\
\text { pagar por salud particular. } \\
\text { Con esfuerzo costean la edu- } \\
\text { cación particular/ particular } \\
\text { subvencionada o universitaria } \\
\text { de sus hijos. }\end{array}$ & $\begin{array}{l}\text { Van a colegios municipaliza- } \\
\text { dos. No tienen posibilidad de } \\
\text { elegir servicios de educación } \\
\text { y salud porque no pueden } \\
\text { pagarlos. }\end{array}$ \\
\hline $\begin{array}{l}\text { Acceso a vivienda/ } \\
\text { localización }\end{array}$ & $\begin{array}{l}\text { Viven en barrios de clase alta } \\
\text { y tienen segunda vivienda. }\end{array}$ & $\begin{array}{l}\text { Viven en nuevas } \\
\text { urbanizaciones. }\end{array}$ & $\begin{array}{l}\text { Viven en viviendas sociales/ } \\
\text { campamentos. }\end{array}$ \\
\hline
\end{tabular}

Fuente: elaboración propia, en base a resultados cualitativos del estudio Anillos 2. 
de diferenciarse de los "otros", aunque sea dentro de la misma clase). Las personas de mayor nivel educacional logran reconocer 5 clases sociales: alta, media-alta, media, media-baja y baja. El grueso de los entrevistados reconoce tres clases sociales: alta, media y baja; mientras que algunas personas de estrato bajo sólo reconocen dos: los ricos y los pobres.

La distinción en términos de ingreso es ejemplificada por los entrevistados al describir las características de cada una de las clases, pues la mayor parte de los descriptores corresponden elementos de consumo no accesibles para la población en general (o al contrario, descriptores que denotan no acceso al consumo). En términos generales, la tabla 2 muestra los descriptores asociados a las distintas clases sociales, considerando los tres grandes grupos distinguidos por la mayor parte de los entrevistados.

Los entrevistados perciben a la sociedad chilena como diversa, trazando una serie de distinciones basadas en capacidad de pago y consumo, que se reflejan, finalmente, en diferentes estilos de vida.

"El otro día conversábamos de, por ejemplo, nosotros prendemos la tele y muestran partes tan lindas, partes tan lindas para las vacaciones que uno ni esperanzas pos. Yo me voy a morir y nunca las voy a conocer. Eso es lo que más de diferente, a lo mejor, tenemos. Lamentablemente, la diferencia en plata. Nosotros vamos a partes más baratitas (risas)" (Mabel, bajo, barrio homogéneo, escuela homogénea).

\section{VISIÓN DE HETEROGENEIDAD DEL ORDEN SOCIAL EN CONTRAPOSICIÓN A LA EXPERIENCIA DE HOMOGENEIDAD DE LAS RUTINAS COTIDIANAS}

A pesar de que las personas identifican sus experiencias cotidianas como homogéneas en términos socioeconómicos, al mismo tiempo señalan que de todos modos conviven con personas distintas. Esto está dado por la importancia asignada a las distinciones socioculturales dentro de cada clase social (especialmente en los sectores medios y altos); esto es específicamente notorio cuando los entrevistados se refieren a los otros apoderados del colegio de sus hijos (donde las distinciones tienen que ver con estructura de la familia, profesión de los padres, aproximación a estilos de crianza de los hijos, etc.).

"O sea, la caricatura de la clase alta es súper marcada. Yo creo que hay de todo. O sea, si tú mirai el mismo barrio donde vivimos nosotros, la caricatura te diría que todos nosotros deberíamos estar viviendo en La Dehesa y por algo estamos acá, y algo hay, que no sé definirlo. Y habrá otra gente que vive más al norte y otra más cerca del barrio El Golf, que alguna inquietud distinta tienen que, si bien por 'la ficha' deberían estar viviendo en Lo Barnechea, están viviendo en otros barrios, pues han escogido otro tipo de vida" (Matías, alto, barrio homogéneo, escuela homogénea). 
Dado que la segmentación intra-clase se experimenta como heterogénea, las personas no significan su experiencia de vida desde la homogeneidad, sino desde la diversidad. Estas distinciones intra-clase obedecen principalmente a criterios culturales dados por: valores a los que adscribe la persona, estilo de vida y consumo (principalmente el eje austeridad/ostentación); lugares de residencia; y colegios a los que manda a sus hijos. Así, en la práctica las personas conciben su vida cotidiana como heterogénea en la medida en que incluyen a personas de su mismo estrato social pero con distintas características socioculturales. Esto lleva a que en muchos casos la homogeneidad social de los espacios que ocupan no sea tematizada.

En tanto, la falta de un vínculo concreto con otro de condición social diferente (más lejana), lleva a que la imagen que se tiene de las personas que pertenecen a una clase social distinta no tenga como base la experiencia cotidiana sino otro tipo de fundamentos. Esto lleva a exagerar o distorsionar los rasgos atribuidos a personas de otra clase social.

"Para mí, lamentablemente el indice de delincuencia es más alto en la clase baja, justamente por cabros que dejan a edad temprana el colegio, hay mucha cesantía, hay mucho consumo de alcohol, mucho consumo de droga" (Cecilia, medio, barrio homogéneo, escuela heterogénea).

De esta forma, se observa que existe una distancia importante entre personas de distintos estratos en términos de su experiencia cotidiana, en la medida en que utilizan espacios distintos. Esto se ve complementado con la percepción de que existen importantes diferencias entre estratos en términos de estilos de vida, ingresos y valores, lo que hace que la convivencia social se perciba, en muchas ocasiones, como imposible e incluso no deseable.

"En cambio aqui hay mucha diferencia cultural, la educación es muy distinta. Entonces qué es lo que pasa, que uno se adapta mucho a personas de clase baja. Encuentro atroz decirlo. Uno tendría demasiados roces y diferencias porque la cultura es muy distinta, porque las costumbres son muy diferentes, y eso en el día a día se hace dificil" (Matías, alto, barrio homogéneo, escuela homogénea).

La diversidad de clases sociales permite que las personas manifiesten que la vida en la ciudad es heterogénea, lo que se constata por medio de la capacidad de distinguir a otros en el espacio social. En general, esta diversidad está asociada a distintos espacios de la ciudad, sobre todo aquellos que poseen establecimientos comerciales o que son zonas de comercio. En general, mientras los espacios residenciales se asocian a homogeneidad, la diversidad está asociada principalmente a aquellos espacios que albergan usos comerciales y a los espacios públicos en que existe mayor control (o normas de convivencias explícitas o implícitas). De este modo, la principal fuente de experiencia que se tiene del otro de distinta condición socioeconómica se da principalmente en espacios públicos 
o comerciales o en los medios de transporte públicos, pero la ausencia de normas explícitas -que genera una incapacidad de predecir el comportamiento-impide la generación del vínculo.

"(refiriéndose al Metro) las otras veces tú te topas con las personas pero te topas no más, en el Metro no te vas a poner a conversar con gente que tú no conoces, no" (Soledad, alto, barrio heterogéneo, escuela heterogénea).

En los espacios públicos, la principal sensación asociada a la presencia del otro es la de incomodidad. Esta incomodidad se asocia a la inconmensurabilidad de la diferencia entre las clases sociales, en la que se pone en discusión la posibilidad de generar un vínculo entre iguales. Desde la mirada de los entrevistados, esta incomodidad da pie para que puedan generarse conflictos sociales ante la ausencia de expectativas recíprocas de comportamiento.

Esta incomodidad se produce en paralelo a un proceso de naturalización de la otredad ${ }^{37}$, en la cual la actitud natural hacia el otro se da en el marco de la indiferencia. De esta forma, el otro pasa a conformar un elemento del entorno, con el cual no existe obligación de interactuar. Al evitar la interacción a través de la indiferencia, se resuelve el sentimiento de incomodidad vinculado a lo impredecible del comportamiento del otro.

“...yo creo que la gente que va a los mall les da lo mismo que la gente se mezcle o no porque viven su mundo no les

37 Saraví, 2008

134 revista invi № 76 / Noviembre 2012 / Volumen Nº 27: 117-145 interesa yo creo, son pocos a los que les interesa qué le pasa al que está al lado" (Iris, bajo, barrio homogéneo, escuela homogénea).

Por otra parte, existen espacios en que la sensación de incomodidad desaparece porque las expectativas están reguladas, es decir, la forma del vínculo social está absolutamente normada. Un importante ejemplo de esto es lo que se produce en el ámbito religioso y de solidaridad, que es uno de los espacios más mencionados por los entrevistados como aquel lugar donde el encuentro con el otro no es incómodo ni conflictivo. En este tipo de espacios, la expectativa se regula en el marco de las "caritas", donde uno da y el otro recibe.

"Cosas de iglesia que van, no sé, yo he estado, me han invitado a encuentros, esas cosas y la verdad es que tú ahi compartes con todo tipo de gente, te toca trabajar con todo tipo de gente y la verdad que las experiencias de vida y las enseñanzas son valorables, bien valorables" (Soledad, alto, barrio heterogéneo, escuela heterogénea).

También constituyen espacios regulados el trabajo, los espacios de salud y en alguna medida la escuela. En estos lugares, el marco normativo favorece la experiencia de contacto con el otro y, por lo tanto, son fundamentales para potenciar los vínculos que permitan redefinir algunos imaginarios marcados por el miedo y la desconfianza hacia el "otro". 
La principal característica que tiene el trabajo es que es uno de los espacios más mencionados a la hora de tener que identificar la mezcla social y, al contrario de los espacios públicos, este es un ámbito que potencia la generación de vínculos sociales, especialmente de sociabilidad entre iguales. Sin embargo, el tipo de vínculo que se establece en el trabajo con personas de distinto estrato social tiende a estar mediado por elementos funcionales propios del tipo de trabajo y de sus formas organizacionales. De esta manera, la sociabilidad se da con más fuerza entre los iguales, en el marco de una relación regulada bajo la expectativa de las prestaciones asociadas al mundo del trabajo.

"(Mis compañeros de trabajo) No son como gente desconocida, pero en el trabajo me junto más con gente similar a mî" (Mabel, bajo, barrio homogéneo, escuela homogénea).

Los espacios de salud están tan normados que la relación más relevante es aquella que se establece con el personal administrativo y de salud, por lo que el criterio de decisión más utilizado, especialmente por aquellos que pueden optar sobre su uso, es la calidad del personal y de las instalaciones. La presencia o ausencia de personas de distinto estrato no sólo no tiene importancia sino que ni siquiera se tematiza, no obstante que se valora:

"me ha tocado ir a los hospitales de repente y me ha tocado ver de todo, de diferentes comunas, me ha tocado ir de repente al hospital, a la posta, de ahí de Estación Central, la posta 3 también y me ha tocado ver que gente chora, gente de clase media, gente que viene de una situación buena y se respetan, el hecho de yo creo estar en un hospital, de ver a la persona que está al lado suyo igual, hace que se respeten, porque ¿quién se va a poner a pelear en un hospital?" (Iris, bajo, barrio homogéneo, escuela homogénea).

Aun así, dado que la calidad de la salud se asocia a la capacidad de pago de las familias, los espacios de salud son percibidos como homogéneos por los entrevistados y más bien segmentados conforme a la capacidad de pago de cada cual.

En el caso de la escuela, las principales limitantes para la convivencia social están dadas por el acceso (la escuela que se escoge está directamente relacionada con la capacidad de pago del hogar), de modo que la mayor parte de los entrevistados reporta escuelas socialmente homogéneas. En aquellos casos donde se tenía la experiencia de heterogeneidad, se percibe que la mezcla no es experimentada como dramática porque hay un marco de igualdad en el trato de los niños (dado por los valores y normas del colegio). En este sentido, la presencia de normas claras y valores bien definidos disminuye las tensiones generadas por la existencia de heterogeneidad social. No obstante, en general estos padres forman vínculos y relaciones con padres de condición socioeconómica similar.

"(Investigador) Y en el caso del colegio, ahí me decías que había grupos distintos, ite relacionas con las personas que se parecen más a ti o con las que se parecen menos? 
Sí, sí, pero por una cuestión de tipo práctica, puede que haya diferencias, pero hay algunos elementos en común que nos pueden unir con algunas personas... no, pero sabes que no, no converso con nadie, sólo con un par porque los genios son más parecidos" (Claudia, medio, barrio heterogéneo, escuela heterogénea).

Es importante señalar que, a pesar de no ser una variable presente en la selección de las escuelas, se valora por parte de los padres un ambiente de diversidad en la crianza de los hijos.

“...En el caso de algunos alumnos se ve que el apoderado trabaja en el colegio y hay alumnos becados por notas; hay gente que en verdad de repente no tienen para los materiales! Pero está igual en el colegio, entonces igual el colegio es súper, prima que los alumnos se relacionen como con todo tipo de personas y no es como iAy, él no paga!" (Carmen, medio, Escuela heterogénea, barrio heterogéneo).

En el caso de los padres que no poseen experiencias de heterogeneidad social, la diversidad viene dada por la percepción de distinciones socioculturales entre los niños de la escuela, sobre todo en los segmentos altos. En general, la ausencia de heterogeneidad social no es un tema relevante para los segmentos altos, ya que precisamente uno de los valores relevantes que posee la escuela es la posibilidad de contar con un ambiente social similar al familiar, que fomente en los niños una educación con valores similares a los que poseen los padres, con un ambiente cultural parecido, además del acceso a redes sociales.
¿Y por qué era para ti importante que compartieran con iguales en el colegio?

Yo creo que es la época en que los niños están más vulnerables, están aprendiendo, están copiando no cierto, están creciendo, están abriendo los ojos, están en todo su desarrollo y todo el cuento... no sé, hay otras costumbres en otras partes, hay otras cosas, entonces tú quieres que más o menos sigan como en lo mismo que tú has inculcado y enseñado en tu casa..." (Carolina, medio, escuela homogénea, barrio heterogéneo).

Los espacios residenciales son los menos heterogéneos, los más abiertos y menos normados. Al igual que en la escuela, la segmentación viene dada principalmente por la capacidad de pago de los hogares (en este caso, de pago por suelo). La homogeneidad es además valorada en términos de preservar un "estilo de vida" o "vida de barrio".

"Un amigo de nosotros alega mucho, les hicieron unos condominios de terrenos más chiquititos, allí antes habia un fundo, sus vecinos eran las palomas, los ratones. Entonces le echaron a perder el barrio, porque claro, no son viviendas sociales claramente... es que mira, hay un tema con la delincuencia con el trafico que es una realidad, o sea, aquí en la villa La Reina es una realidad, mi nana vive y me dice que es pelúo (Carolina, alto, barrio homogéneo, escuela homogénea).

Lo que se va produciendo es que en aquellos espacios percibidos como relevantes, tales como el barrio y la escuela, la estructura de oportunidades se segmenta mediante el pago de servicios y por lo tanto, se favorece una sociabilidad con el 
otro en espacios homogéneos. Las experiencias de heterogeneidad están dadas en espacios que se consideran menos significativos en la experiencia individual (principalmente malls y otros espacios públicos como grandes parques), que permiten, por una parte, ser más indiferente a la presencia de un otro diferente, y por otra, tener la tranquilidad de que existe un marco normativo que regula las posibles interacciones (como en el caso del trabajo, la escuela o la iglesia).

No obstante, es importante señalar que, en términos generales, las personas perciben que la experiencia más cotidiana de mezcla se da entre la clase alta y la clase media, en donde el compartir los espacios sociales es menos conflictivo, más natural, y que el verdadero ausente en la experiencia cotidiana es la clase baja, que utiliza espacios totalmente distintos (educación y salud pública, vivienda social y poca presencia en espacios de consumo).

\section{DISPOSICIÓN HACIA LA HOMOGENEIDAD/ HETEROGENEIDAD}

De los relatos de los entrevistados se desprende que la heterogeneidad en sí misma no es un valor explícitamente buscado. En general, las personas que transitan por lugares heterogéneos han realizado la elección por otras razones distintas a la heterogeneidad. De esta forma, la disposición a la convivencia no es un valor en sí mismo, sino que se interpreta como el resultado de otras elecciones diferentes. Este resultado es aplicable para las personas de distintos niveles socioeconómicos.

"Investigador) Entonces, cuando tú encontraste tu casa o buscaste el colegio de tu hija, ifue importante para ti que fuera homogéneo o heterogéneo el lugar donde quedaba socialmente o fue algo que salió por casualidad?

"No es que haya estado buscando heterogeneidad, estaba buscando seguridad y yo creo que eso sí está asociado a ese tipo de entorno, es que estaba pensando que fuera un lugar donde podría salir a caminar con mi hija a la esquina, digamos no le llegara un balazo por ahî" (Claudia, medio, barrio heterogéneo, escuela heterogénea).

La disposición a la convivencia con otro distinto está cruzada por dos temas. Por una parte, la existencia de una noción de justicia asociada a la igualdad de oportunidades, y por otra parte, por la imagen que se tiene del otro.

En primer lugar, el principal motivo que genera cercanía a la idea de espacios más heterogéneos es que medidas de este tipo permitirían que personas de distinto estrato tuvieran un igual acceso a oportunidades (educación de calidad, cercanía a servicios y comercio, espacios públicos de calidad, etc.).

De este modo, la disposición a la convivencia con otro se ve afectada por el valor que las personas entregan a la socialización del bienestar. En este sentido, en estratos medios y altos hay apertura a la convivencia con el otro de condición socioeconómica menor en los casos en que se considera que no hay justicia social, por lo tanto, es importante 
entregar al otro un mínimo social para que pueda tener un marco para emprender. Sin embargo, es importante señalar que las personas no consideran que la solución debiera ser el aumento de la heterogeneidad de los espacios que ellos habitan, sino más bien una subida del estándar de las prestaciones que el Estado ofrece a los sectores populares (calidad de la educación y la salud, calidad de la vivienda y barrios).

"Yo me he atendido en hospitales en reiteradas ocasiones y en lo privado. Conozco además el sistema de fuerzas armadas, y creo que es esencial que la gente tenga acceso a los tratamientos, medicamentos y cosas de recuperación... creo que tiene que ser una salud pública, no habría que pagar, son derechos básicos" (Carmen, medio, barrio heterogéneo, escuela heterogénea).

Los estratos bajos perciben mayores beneficios asociados a la convivencia, tanto en términos de contactos como en la calidad de las prestaciones y servicios a los que podrían acceder. Asimismo, perciben que disminuirían los problemas de criminalidad al vivir en un "mejor barrio".

En segundo lugar, el principal temor que se tiene (por parte de estratos medios y altos) a compartir el espacio con personas de menor nivel socioeconómico es la imagen que se tiene de ellos, ya que se los asocia a costumbres completamente distintas (lo que dificulta pensar en compartir la escuela) y a actividades delictuales (lo que los vuelve indeseables como vecinos), por lo que se asume que su llegada sería conflictiva.

Investigador: "Si llegara un condominio de clase media, sería como una villa de La Florida o Maipú".

Entrevistado: "No, o sea, es más como el tema de la droga, de la delincuencia, es como eso. Eso es lo que me preocupa" (Carmen, medio, escuela heterogénea, barrio heterogéneo).

En el caso de los sectores bajos, el principal obstáculo a la convivencia con personas de sectores más altos, se asocia a ser sujetos de discriminación o de un trato desigual y que sus hijos desarrollen expectativas que más tarde no podrán cumplir. Más allá de que las personas hayan tenido experiencias efectivas de discriminación, la posibilidad de someterse a esa experiencia inhibe el contacto social.

"Que los puedan discriminar porque siempre se da en las conversaciones de los papás... el papá de él trabaja en muebles y a lo mejor el otro no. No sé. Eso me daría un poco de miedo" (Mabel, bajo, barrio homogéneo, escuela homogénea).

La disposición a la convivencia con el otro es diferente de acuerdo al nivel socioeconómico de las personas. En el caso de los segmentos altos y medios, la primera reacción frente a la posibilidad de convivencia, muestra el bajo beneficio que tendría para ellos convivir con personas más pobres. De esta forma, los pobres no tendrían un valor concreto que aportar para su vida cotidiana y serían 
ellos los más beneficiados por la convivencia. Por otra parte, el mayor valor que se otorga a la convivencia por parte de aquellos hogares de condición socioeconómica baja es la posibilidad de acceder a servicios y prestaciones derivadas de la proximidad (mejora de sus oportunidades de vida). De esta manera, la expectativa para formar vínculos está fuertemente permeada por la funcionalidad del contacto.

Adicionalmente a lo ya señalado, se generan algunos beneficios y/o dificultades específicos cuando se pone en el escenario de compartir el barrio o la escuela a personas de distinta condición social.

En el caso de la escuela, los principales beneficios de la heterogeneidad social, de acuerdo a la percepción de los entrevistados de estratos medios y altos, son que sus hijos aprendan a vivir en la diversidad y tengan noción de lo que ocurre en otras realidades. Esto queda, sin embargo, más bien en el discurso, en la medida en que en general el criterio de heterogeneidad social no es utilizado al momento de escoger el establecimiento al que se enviará a los hijos.

"O sea, yo creo que es beneficio pa'l que va y también pa'l resto de los cabros que pueden ver otras realidades, hoy por nuestros hijos están viviendo un mundo de ricos y famosos y que no tienen una real conexión con la pobreza". (Carolina, alto, escuela homogénea, barrio homogéneo).
En el caso de los entrevistados de estratos bajos, se declara que el principal beneficio de la heterogeneidad social de las escuelas es que, tal como señalan los entrevistados de estratos altos y medios, se aprendería a vivir en diversidad y las personas de estratos altos aprenderían otros valores importantes, como el sacrificio. Por otra parte, para ellos implicaría acceder a educación de mayor calidad (en la medida en que se asocia la llegada de personas de mayor nivel socioeconómico a un aumento de la calidad) y a personas con mayor nivel cultural, de las que se puede aprender.

"(Investigador) ¿Y tú crees que podrías aprender algo de esa persona de clase más alta?"

"Sí, yo creo que sí.

(Investigador) "¿Qué cosas por ejemplo?"

"Es que, en general, ellos son súper estudiados po' y yo creo que podría aprender más como eso" (Mabel, bajo, barrio homogéneo, escuela homogénea).

Las dificultades asociadas a la heterogeneidad social en la escuela cambian drásticamente según el estrato al que se consulte. En el caso de los estratos medios y altos, el principal temor es que los niños de menor nivel socioeconómico les contagien malas costumbres a sus hijos. En el caso de los estratos bajos, los temores están asociados a que los niños desarrollen expectativas que como padres no podrán cumplir, y a la vergüenza (y temor a la 
TABLA 3: BENEFICIOS Y AMENAZAS DE LA HETEROGENEIDAD EN LA ESCUELA, DE ACUERDO AL NIVEL SOCIOECONÓMICO.

\begin{tabular}{|c|c|c|}
\hline & $\begin{array}{l}\text { Estratos altos/medios con } \\
\text { respecto al estrato bajo }\end{array}$ & Estratos bajos con respecto a los estratos medios y altos \\
\hline Beneficios & $\begin{array}{l}\text { Aprender de otras realidades } \\
\text { (discursivo, en la práctica no } \\
\text { implica movilizar la necesidad } \\
\text { de generarvínculos con otros) } \\
\text { Aprender a vivir en } \\
\text { diversidad. }\end{array}$ & $\begin{array}{l}\text { Aprender de los otros y poder acceder a personas con mayor nivel cultural } \\
\text { Aprender a vivir en diversidad. } \\
\text { Acceder a mejor educación (en la escuela se asume que por llegar gente } \\
\text { de clase alta ésta será mejor). } \\
\text { La gente de clase alta aprendería otros valores importantes asociados } \\
\text { con la clase baja (valor del sacrificio). }\end{array}$ \\
\hline Amenazas & $\begin{array}{l}\text { Aprendizaje de malas } \\
\text { costumbres. }\end{array}$ & $\begin{array}{l}\text { Vergüenza de que los amigos de los hijos vean cómo viven. } \\
\text { Los niños podrían aspirar a cosas que no les pueden dar. }\end{array}$ \\
\hline
\end{tabular}

\section{TABLA 4: BENEFICIOS Y AMENAZAS DE LA HETEROGENEIDAD EN EL BARRIO, DE ACUERDO AL NIVEL SOCIOECONÓMICO.}

\begin{tabular}{|c|c|c|}
\hline & Estratos altos/medios con respecto al estrato bajo & $\begin{array}{l}\text { Estratos bajos con respecto a los estratos medios } \\
\text { y altos }\end{array}$ \\
\hline Beneficios & $\begin{array}{l}\text { Aprender de otras realidades (discursivo, en la prác- } \\
\text { tica no implica movilizar la necesidad de generar } \\
\text { vínculos con otros). } \\
\text { Beneficios funcionales de acceso a prestaciones y } \\
\text { servicios por cercanía de viviendas. }\end{array}$ & $\begin{array}{l}\text { Aprender de los otros y poder acceder a personas } \\
\text { con mayor nivel cultural. } \\
\text { Aprender a vivir en diversidad. } \\
\text { Beneficios funcionales por prestación de servicios } \\
\text { por cercanía de viviendas. } \\
\text { La gente de clasealta aprendería otrosvaloresimpor- } \\
\text { tantes asociados con la clase baja (valor del sacrificio). } \\
\text { Menos criminalidad y droga por la llegada de per- } \\
\text { sonas de estrato alto. } \\
\text { Mejoramiento de la estética del barrio por llegada } \\
\text { de personas de clase alta. }\end{array}$ \\
\hline Amenazas & $\begin{array}{l}\text { Aprendizaje de malas costumbres. } \\
\text { Pérdida de plusvalía de las viviendas. } \\
\text { Pérdida de tranquilidad de la vida de barrio. } \\
\text { Pérdida de la estética del barrio: aumento de basu- } \\
\text { ra, grafitis y ruidos. } \\
\text { Pérdida de seguridad, y aumento de criminalidad ydroga. }\end{array}$ & Discriminación y falta de respeto al estilo de vida. \\
\hline
\end{tabular}


discriminación) que les da el que los compañeros de sus hijos vean sus condiciones (principalmente de vivienda).

En el caso de los beneficios y problemas asociados a la heterogeneidad en el barrio, se mantienen los detectados para la escuela y se añaden otros nuevos.

Sobre los beneficios que los estratos altos y medios identifican, se encuentra nuevamente el aprender de otras realidades y se añade el reconocimiento respecto al aporte en beneficios funcionales que una medida así tendría para las personas de estrato bajo. En el caso de las personas de estrato bajo, a los beneficios de aprendizaje de otros con mayor nivel cultural y capacidad de vivir en diversidad, se añaden los beneficios funcionales de vivir en un barrio con mayor nivel socioeconómico, tanto en términos de servicios como en relación a la belleza del barrio y la menor exposición al crimen. Se señala, además, que las personas de estratos altos y medios aprenderían de otros valores, más presentes en los estratos bajos, como el sacrificio.

"Yo creo que sí se vería el cambio, a lo mejor no andaría tanto niño en la calle a esa hora, tipo 2 de la noche o de repente no andarían tanto cabros chicos. Yo creo que se eliminarían los drogadictos, yo creo que si porque la gente de media va a querer que haiga [sic] más seguridad, cuidar las cosas de uno" (Iris, bajo, escuela homogénea, barrio homogéneo).
Acerca de las amenazas identificadas, en los estratos altos se añade, además de la posibilidad de aprendizaje de malas costumbres señalado respecto a la escuela, la pérdida de plusvalía de las viviendas, y deterioro tanto de la tranquilidad y seguridad del barrio como de sus características estéticas y de rutinas cotidianas, lo que afectaría la "vida de barrio" que se desarrolla en el lugar. Los entrevistados de estratos bajos, en cambio, sólo identifican la discriminación y la posible falta de respeto a su estilo de vida como posible amenaza.

"Pero que estén ubicadas donde se pueda y donde no te afecte tu casa. No por el vivir, porque yo en algún momento me voy a querer ir y si me ponen un bloque al lado con 150 familias, nadie me la va a querer comprar" (Carolina, alto, barrio homogéneo, escuela homogénea).

En síntesis, si bien no existe una convivencia deliberada entre las diferentes clases sociales, en los discursos de los entrevistados aparecen algunas condiciones que posibilitarían dicha convivencia. Estas condiciones no son las mismas para los diferentes espacios, sin embargo, en los discursos de los entrevistados aparecen dos formas asociadas a esta convivencia:

a) Características físicas de los espacios: la convivencia entre clases sociales se puede potenciar siempre y cuando los espacios físicos no se echen a perder, no se vuelvan inseguros o las casas no pierdan su plusvalía. En este sentido, la condición 
para la convivencia está puesta en que se asegure la preservación física del entorno.

b) Marco normativo: otra condición para potenciar la convivencia entre clases está dado por contar con una regulación externa que enmarque los términos de la relación. Por ejemplo, en el caso de la escuela, contar con normas claras que regulen los valores de la convivencia, sin transar la calidad de la educación; o en el caso de espacios públicos, el mayor control policial que resguarde la seguridad.

Estas condiciones para la convivencia dan cuenta de que aún existe un margen para convivir con otro de diferente clase social y que la heterogeneidad en sí no es desechada, sino que se hace necesario contar con ciertos elementos del entorno que la posibiliten.

\section{Conclusiones y reflexiones}

De las entrevistas se desprende que hoy en día existe una importante segmentación estructural de los espacios sociales (dada porque el acceso a dichos espacios está mediado por el pago), lo que lleva a la conformación de barrios, escuelas y otros espacios como lugares socialmente homogéneos, en que no existe posibilidad de contacto con otro de condición socioeconómica distinta. En este sentido, se ha producido una división del espacio

142 revista invi № 76 / Noviembre 2012 / Volumen N ${ }^{0} 27: 117-145$ como un correlato a la estratificación de clases de la sociedad ${ }^{38}$.

Esta segmentación de la estructura de oportunidades, que además tiene un correlato en la calidad de los espacios (quienes acceden a mejores barrios, mejores escuelas, etc., son quienes tienen mayores ingresos), está legitimada desde la movilidad social. Si bien la distribución se percibe como injusta, quienes están en las posiciones menos privilegiadas piensan que sus hijos podrán acceder a los privilegios.

La movilidad supone una experiencia de sociedad que se abre a las oportunidades, que si bien han sido reales para muchos chilenos (ascenso social respecto al nivel socioeconómico de los padres, acceso a la vivienda propia, acceso de los hijos a la educación superior y acceso al consumo en general), es una experiencia radicalmente distinta a las posibilidades que existían dentro de la sociedad chilena de la primera parte del siglo XX, en donde las oportunidades estuvieron más asociadas a la adscripción y la restricción a la mezcla fue explícita.

"Mi mamá me cuenta que la familia de mi abuela era así. No se podían mezclar las clases. O sea, la clase alta no se podía juntar con los trabajadores. Pasó con mi abuela que se casó con mi abuelo que trabajaba en una hacienda y a mi abuela la desheredaron por la misma situación. Aun así mi abuela se casó y como que la desterraron" (Tabita, bajo, barrio heterogéneo, escuela heterogénea).

38 Saraví, 2008. 
De esta forma, lo que se está produciendo a nivel cultural es la formación de un núcleo valórico que legitima la distribución de las oportunidades sociales (y por ende, su traducción en espacios con ciertas características) desde el esfuerzo individual y dentro de un escenario de igualdad de oportunidades.

Lo que aparece problemático no es la segmentación socioeconómica de los espacios, sino la desigualdad de oportunidades. La heterogeneidad social de los espacios no es vista como un valor en sí. El problema de los espacios heterogéneos es que algunos hogares no acceden a los servicios y prestaciones que debieran. En la concepción de los entrevistados, se trata más de un problema de equidad que de convivencia.

Del relato de los entrevistados, emerge con fuerza la falta de vínculo efectivo con el otro de condición socioeconómica distinta. Este debilitamiento del vínculo tiene importantes consecuencias asociadas a la valoración del otro. En la medida que se debilita la experiencia cotidiana del otro, lo que se impone para configurar la otredad es un imaginario permeado por la desconfianza y el desconocimiento de ese otro. Y es en base a este imaginario, que se decide la disposición a la participación en espacios mezclados socialmente.
De ahí que en términos de convivencia social, los espacios institucionales regulados adquieran importancia en la medida que logran normar la expectativa del otro, de manera que su comportamiento se ajuste de alguna forma a la normativa, volviéndolo predecible. Habría que indagar en qué medida la experiencia del otro se puede construir en estos espacios, identificando las barreras y disposiciones que existen en torno a ellos.

Es importante señalar que si bien la ausencia de experiencia del otro nos lleva a construir una sociedad descohesionada o fragmentada, la experiencia del otro no necesariamente debería producir una sociedad cohesionada, ya que dicha experiencia puede ser también conflictiva. Una mala experiencia del otro puede incidir en la falta de disposición a la convivencia con otro, ya que se tienden a confirmar los prejuicios con las vivencias efectivas.

Queda pendiente en esta investigación, discutir acerca de distintos tipos de cohesión que se pueden configurar a partir de diferentes experiencias de los sujetos. No obstante, esta tipología podría ser abordada con metodologías distintas que permitiesen caracterizar mejor las experiencias de los individuos y cómo ellas afectan su disposición hacia la convivencia con otros. 


\section{Bibliografía}

BROOKS-GUNN, Jeanne. Stressful events in adolescence. En: FIELD, Tiffany. Stress and coping in infancy and childhood. New Jersey, Estados Unidos, Lawrence Erlbaumm Associates, Inc. 1992. p. 119-139. ISBN 0-8058-0944-9.

CONSEJO Asesor Presidencial Trabajo y Equidad. Informe final: hacia un Chile más justo: trabajo, salario, competitividad y equidad social. Santiago, Chile, El Consejo. 2008. 250 p.

FLORES, Carolina. Residential segregation and the geography of opportunities: a spatial analysis of heterogeneity and spillovers in education. Austin, Estados Unidos, LBJ School of Public Affairs The University of Texas. 2008.

HARVEY, David. Social justice and the city. Baltimore, Estados Unidos, The Johns Hopkins University Press. 1973. 336 p. ISBN 080181524X.

HOPENHAYN, Martin. Cohesión social: una perspectiva en proceso de elaboración. En: SOJO, Ana y UTHOFF, Andras. Cohesión social en América Latina y el Caribe: una revisión perentoria de algunas dimensiones. Santiago, Cepal. 2007. p. 1-246. Colección documento de proyecto.

JENCKS, Christopher y MAYER, Susan. The social consequences of growing up in a poor neighborhood. En: LYNN, Laurence E. Inner-city poverty in the United States. Washington D.C., Estados Unidos, National Academy Press. 1990. p. 111186. ISBN 0-309-04279-8.

144 revista invi № 76 / Noviembre 2012 / Volumen N N 27: 117-145
KAZTMAN, Rubén. Seducidos y abandonados: el aislamiento social de los pobres urbanos. [En línea]. Revista de la Cepal. (75): 171-189, diciembre 2001. ISSN 0251-0257. Disponible en: http://www. eclac.cl/cgi-bin/getProd.asp?xml=/revista/noticias/articuloCEPAL/6/19326/P19326.xml\&xsl=/ revista/tpl/p39f.xsl\&base=/tpl/top-bottom.xslt\#.

KESSLER, Gabriel. El sentimiento de inseguridad: sociología del temor al delito. Buenos Aires, Argentina, Siglo XXI. 2009. 287 p. ISBN 9876290975.

LEFEBVRE, Henri. El derecho a la ciudad. Barcelona, España, Península. 1969. 169 p. ISBN 84-297-0916-9.

MÁRQUEZ, Francisca. Imaginarios urbanos en el Gran Santiago: huellas de una metamorfosis. [En línea]. Eure. 99(33): 79-88, agosto de 2007. ISSN 07176236. Disponible en: http://dx.doi.org/10.4067/ S0250-71612007000200007.

MEDINA ECHAVARRíA, José. Consideraciones sociológicas sobre el desarrollo económico de América Latina. Santiago de Chile, Editorial Universitaria. 1976. 218 p.

SABATINI, Francisco, CÁCERES, Gonzalo y CERDA, Jorge. Segregación residencial en las principales ciudades chilenas: tendencias de las tres últimas décadas y cursos de acción. [En línea]. Eure. 27(82): 21-42, diciembre 2001. ISSN 07176236. Disponible en: http://dx.doi.org/10.4067/ S0250-71612001008200002.

SABATINI, Francisco; SALCEDO, Rodrigo; WORMALD, Guillermo y CÁCERES, Gonzalo. Tendencias de la

ARTÍCULO: Cultura de cohesión e integración en las ciudades chilenas / Guillermo Wormald, Carolina Flores, Francisco Sabatini, María Paz Trebilcock y Alejandra Rasse Figueroa 
segregación en las principales ciudades chilenas: análisis censal 1982-2002. Santiago de Chile, Ediciones INE. 2010. 186 p. ISBN 9561411105.

SARAVÍ, Gonzalo. Mundos aislados: segregación urbana y desigualdad en la Ciudad de México. [En línea]. Eure. 34(113): 93-110, diciembre 2008. ISSN 0717-6236. Disponible en: http://dx.doi. org/10.4067/S0250-71612008000300005.

SENNET, Richard. El declive del hombre público. Barcelona, España, Península. 1978. 433 p. ISBN 8429714456.

SLATER, Don y TONKINS, Fran. Market society. Cambridge, Inglaterra, Blackwell Publishers. 2001. 233 p. ISBN 0745620272.

TORCHE, Florencia y WORMALD, Guillermo. Estratificación y movilidad social en Chile: entre la adscripción y el logro. Santiago, Chile, CEPAL, División de Desarrollo Social. 2004. 85 p. ISBN 92-1-322604-7.
TORCHE, Florencia. Unequal but fluid: social mobility in Chile in comparative perspective. [En línea]. American Sociological Review. 70(3): 422-450, junio 2005. ISSN 00031224. Disponible en: http:// dx.doi.org/10.1177/000312240507000304.

UNIVERSIDAD Católica y ADIMARK. Encuesta Nacional Bicentenario. [En línea]. 2009. [Fecha de consulta: 10 de agosto de 2010] Disponible en: http:// www7.uc.cl/encuestabicentenario/.

VALENZUELA, Eduardo. Desorganización, solidaridad y movilidad. En: VALENZUELA, Eduardo; SCHWARTZMAN, Simón; VALENZUELA, J. Samuel; SCULLY, Timothy; SOMMA, Nicolás y BIEHL, Andrés. Vínculos, creencias e ilusiones: la cohesión social de los latinoamericanos. Santiago, Chile, Uqbar. 2008. p. 17-60. ISBN 978-956-8601-17-1.

WILSON, William J. When work disappears: the world of the new urban poor. New York, Estados Unidos, Vintage Books. 1997. 322 p. ISBN 0-679-72417-6.

revista invi ํำ 76 / Noviembre 2012 / Volumen N ${ }^{0}$ 27: 117-145 145 